\title{
Assessing knowledge creation process: A case of the National Disaster Management Agency of Gambia
}

\author{
Mbassi Saneh ${ }^{1}$, Alfi Rahman $^{2,3^{*}}$, and Khairul Munadi ${ }^{3}$ \\ ${ }^{1}$ Graduate Program in Disaster Science (MIK) Universitas Syiah Kuala, Darussalam, Banda Aceh, Indonesia \\ ${ }^{2}$ Pusat Riset Ilmu Sosial dan Budaya (PRISB), Universitas Syiah Kuala, Darussalam, Banda Aceh, Indonesia \\ ${ }^{3}$ Tsunami and Disaster Mitigation Research Center (TDMRC), Universitas Syiah Kuala, Darussalam, Banda Aceh, Indonesia
}

\begin{abstract}
The Disaster Risk Management policy and strategy of Gambia's National Disaster Management Agency (NDMA) have technical challenges and require knowledge creation to enhance capacities to lessen the adverse effect of risks and probabilities of disasters in The Gambia. Also, limited or no resources and leadership support to knowledge creation. The research aimed to assess the current condition of knowledge creation process in NDMA on socialisation-sharing experiences from tacit knowledge, externalisationvoicing tacit to explicit, combination-structuring explicit, and internalisation-expressing explicit to tacit (SECI) and role of leadership in the knowledge creation process. Detailed interviews, questionnaires, and WhatsApp call employed with four questions targeted five staff informants from NDMA and eight partner institutions with one informant from each. The research adopted a qualitative case study and content analysis to analyse the data using Voyant tools looking at the subject and concept. The research found that SECI has unearthed NDMA strengths, weaknesses, opportunities, threats, and leadership bureaucracy coupled with fear factors to build capacities leading to coup d'état for positions. Therefore, SECI has been unnoticed and uncoordinatedly implemented from "Happy Corner" with experiential knowledge and the National Platform for disaster risk reduction (DRR).
\end{abstract}

\section{Introduction}

The National Disaster Management Agency (NDMA) of Gambia was established by an Act of parliament 2008 and mandated to coordinate disaster management issues in The Gambia with Strategy and Policy frameworks. It has the political will from the highest office of the Vice President as the overall supervisor and the chairperson of the disaster governing council, which comprises all government ministries, private sectors, academic institutions, Red Cross Society, religious leaders, NGOs, civil society, media, and UN agencies. The dayto-day administrative affairs are under the purview of the Executive Director as liaison to multifaceted institutions for the implementation of disaster activities in building a resilient country. The agency has decentralised structures at regional and municipal, district, and village levels in the country's seven administrative regions: the Governors and Mayors, Chiefs, and Alkalos (village head) as administrative chairpersons.

With established structures and political will, NDMA performance has many challenges, especially technical capacities in Disaster Risk Management and Disaster Risk Reduction (DRM/DRR). The performances are required knowledge for continued innovation, education, and understanding of emerging hazards which is weak in NDMA. Also, frequent leadership changes, limited human and financial resources, and policy and governing gap to act as mentioned in the Act 2008 [1]. The 2012 and 2017 capacity and needs assessment highlighted these challenges in NDMA and partner's roles and responsibilities for operations not fully implemented due to inadequate knowledge, skills, and experiences. Thus, due to the knowledge capacities gap in individual, institutional, environmental, and policy creations, utilisation and retention need to be upgraded by creating new opportunities and building blocks for the creation of new knowledge for the personal and institutional growth of technicians trained and aware of the past, present, and probable future of DRM/DRR. And this will improve operations and implementations at all levels [2].

Therefore, a report on capacity and needs assessment and the revised strategy and policy seriously mentioned NDMA and its partner capacity to be continuously strengthened to implement DRM/DRR. In recent years, the Gambia has been exposed to hazards and vulnerable because the country is geographically right of the River Gambia flood plain and makes the country experiencing yearly flooding. The Gambia is encountering both hazards-natural and human-induced. It affects thousands of individuals yearly, such as flooding, windstorms, disease outbreaks, deforestation, oil spillage, bush and domestic fires, droughts, pest invasion, urban settlements, industrial pollution, toxic waste, and accidents. And of the recent political impasse in the

${ }^{*}$ Corresponding author: alfi.rahman@unsyiah.ac.id 
2016 presidential election brought political conflict. The NDMA couldn't coordinate due to the fear factor under the purview of the president's office during the period.

The intensity and the scale of disasters defer in the urban and rural areas and paralysed community services, loss of lives and environmental damages, infrastructures, and the incomes of the people and country. Besides, Disasters are unexpected, unpredicted, and surprising events with a devastating intensity which is stressful on communities and people. Therefore, the know-how (skills) on knowledge and creation is incumbent on every institution reducing the impact of disasters through continuous knowledge creation on unforeseen hazards. To help protect safer and build resilient communities and the country from the effects. Knowing and knowledge of skills and experiences of employees is paramount via continuous knowledge creation $[3,4]$. Negligence to the consideration of such can lead to inefficacy and failure. Therefore, investing in human and technological capital in achieving NDMA's vision is a worthy cause. DRM requires continuous knowledge creation for a sustainable environment, society, and development [5]. Hyogo Frameworks for Action 2005-2015, Sendai Framework 2015-2030, and ECOWAS Policy for DRR 2006 are also highlighted as above. Both concepts require a holistic approach with appropriate knowledge for the implementation of DRM/DRR. Experiences and training exercises on knowledge learned before disaster risk reduction are paramount to share and transfer to bridge the institution's knowledge gap. In a nutshell, DRM/DRR matters are multi-sectoral and multidimensional, which call for absolute mainstreaming of all stakeholders for sustainable development [3].

The institution challenged by "knowledge society" and knowledge (know-how) for effective performance belongs to a person equipped with knowledge [6]. Therefore, knowledge creation is about socialisation being the root of a tree that branches to the SECI process to give detailed information worthy when meeting with people at a place. Further stated knowledge is tacit and explicit interaction: inbuilt thoughts that form the understanding when communicated with a clear and meaningful explanation to audiences [7].

The knowledge creation (KC) process is unstructured and unmethodical when introduced to institutions, groups, and individuals for action. Because it requires creativity to modify the ideas to suit the needs for application [5]. To enable the KC process, there should be socialisation and connection between tacit and explicit knowledge, followed by knowledge assets experiential, conceptual, systemic, and routine to facilitate the $\mathrm{KC}$ process of an institution. $\mathrm{KC}$ is convenient by the context in which and manner the task is done by individuals and groups using knowledge as the driving force to create new knowledge. Cognisant that creating new knowledge cannot be divorced from tacit and explicit [7]. KC process theory includes
Socialisation, Externalisation, Combination, and Internalisation (SECI) which spearhead activities that enable the creation, transfer, and utilisation of knowledge captured from the $\mathrm{Ba}$. $\mathrm{Ba}$ is an open place where people meet, interact, and discuss.

Thus, new knowledge created, shared, and used either analogue (tacit) or the combination (explicit) pass through Information Technology (IT) infrastructure, in which the context and content must be clear to your partner [7].

Currently, there is growing interest in disaster $\mathrm{KC}$ and sharing from lessons and best practices of DRM/DRR strategies to reduce the impact of disasters, informing disaster risk management and disaster risk reduction [3,5]. For this reason, $\mathrm{KC}$ is relevant in the $\mathrm{DRM} / \mathrm{DRR}$ strategy, whereby institutions and individuals $\mathrm{KC}$ have different approaches when introduced for the implementation process. In this sense, $\mathrm{KC}$ cannot be over sighted in the DRM/DRR strategy to elevate the current status of the NDMA. Continuous promotion of knowledge and creation for the performance of employees and the installation of IT infrastructures software monitoring the trend of disasters in the country and knowledge as the knowledge management system (KMs).

Achieving the vision of NDMA DRM/DRR strategy, knowledge management is an integral part of increasing the potential of existing knowledge and transforming new knowledge into action through Information Communication Technology (ICT). This process is easy, quick, accessible, and reliable for communicating and monitoring disaster risk management and disaster risk reduction and the knowledge asset of an institution.

The aims of this study are to assess the current condition of the $\mathrm{KC}$ process on SECI model and to highlight the role of leadership in the $\mathrm{KC}$ process in NDMA.

\section{Knowledge creation}

\subsection{Knowledge creation theory}

\subsubsection{Knowledge}

Leadership has little comprehension of how to create and manage knowledge amicably. Institutions challenged by "knowledge society" and knowledge (know-how) for effective performance belong to a person equipped with knowledge. Therefore, knowledge is about socialisation being the root of a tree that branches to the SECI process to give detailed information worthy for the purpose at a particular time of meeting with people anywhere. Nonaka et al. (2000) further stated there are two types of knowledge - tacit and explicit interaction are inbuilt thoughts that form the understanding when communicated with a clear and meaningful explanation to audiences [6]. Knowledge is 
epistemological that transforms tacit and explicit for action. Ontological is the content and context of the subject reality from socialisation from individual knowledge to group [5]. Knowledge is a process within the human that is "justifying personal belief" towards the "truth" and evidence (s) for people to believe [6]. Knowledge links to performances and outputs via learning to attain, use, and value-added.

Knowledge translates to "know-what, know-how, know-who, and know-why" [8]. For example, the perception of the institution's knowledge is skills and attitudes, while strategic perception defines knowledge as institution resources to attain goals. Knowledge is produced from processed data to generate information [3]. The analytical knowledge block hierarchy portrayed the flow of steps from data, information, knowledge, wisdom, and decisions. Knowledge is often confused with information. Knowledge at times is defined as an IT database system that processes data and information stored for retrieval. Contrary, data is raw without context to give you a meaning for interpretation. Example numbers or reports that signify nothing, but if the context and the value are visualised, you can have the content of the information processed to give a simple, meaningful, accurate, realistic, and timely description for dissemination or dissemination application. Example who, where, when, and what, which turns to knowledge by analysing the information. Knowledge is the information you analyse and use to achieve meaning. Information is transformed into knowledge for action and can be transferable. Wisdom is the application of knowledge for decision-making in any given situation.

In the same vein, resilience is compounded with utilising existing knowledge to produce new knowledge by investing in human and technology resources with the provision of data, information, know-what, and know-how worth consideration for implementing the DRM/DRR. To achieve the goal of resilience, resources must be available including, knowledge to withstand circumstances [9-12]. This cannot be in the absence of knowledge as institutional resources in a competitive environment. Knowledge is created through physical, technological, and human resources [1]. The knowledge produced resources and ploughed to increase physical, technical, and human capital to give supplementary resources in disaster risk management and disaster risk reduction (create new knowledge) to improve decision making. The figure below shows knowledge ladder flow in DRM/DRR for resilience building.
Knowledge Ladder

People's resilience towards disaster is strongly related to the knowledge that triggered informed decision or actions

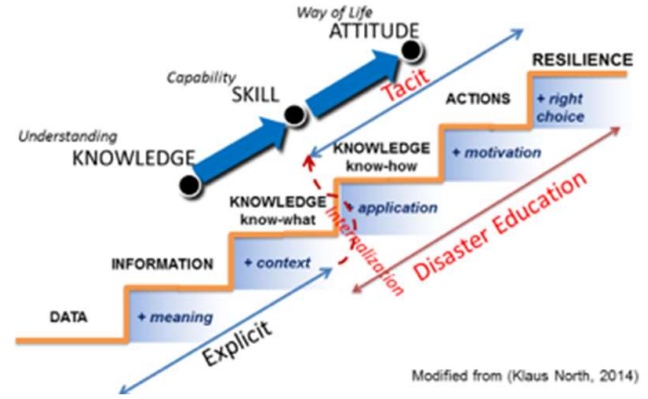

Fig. 1. Knowledge hierarchy Klaus North, (2014) [13]

Knowledge hierarchy corresponding below.

- Know-what defines the action to comprehend knowledge by learning, extensive training for certification, and analysing to improve the institution's quality (Cognitive knowledge).

- Know-how is the ability to practice and apply knowledge gained from the theory. That is capability and competence for the implementation of the knowledge for problem-solving.

- Know-why is the action that motivates decisionmaking for resilience building from the information received. The same as understanding the system of the cause and effect of underlying-problem.

- Ba is also defined as data that provides information, knowledge, and action to create new knowledge of wisdom and decision.

\subsubsection{The SECI process}

Socialisation is from tacit-to-tacit knowledge from interactions with individuals or groups of people to share experiences whereby you capture and disseminate new knowledge. During this time, communication is difficult for people to understand because the information given is chaotic due to the unstructured presentation. The process can be shared through actions, procedures, routines, practice, and ideas from the open place [7].

Externalisation tacit knowledge is interpreted in a comprehensive method to be understood. It is communicated with a group of people from tacit to explicit. The two externalisation factors are (1) Expressing tacit knowledge to explicit from technical ideas and images that form meaningful words. For example, dialogue "listening and contributing to the benefits of all informants." (2) Interpreting tacit clearly through rational thinking to capture the ideas shared [7].

Combination is explicit to explicit knowledge interaction. Explicit converted to systematic and context-specific to produce training manual, article, or report for use. This knowledge is acquired both inside 
and outside of the institution. It accumulates to form new knowledge from a combination of different sources to a new context. The knowledge generated from externalisation is transformed into digital or correspondent material to capture, disseminate, and edit. The materials are shareable and used for communication [7].

Internalisation is to convert explicit to tacit through institutions and individual knowledge during training, learn-on-the-job, and simulation exercise. Knowledge creation is a cycle within the SECI process. Internalisation is the technical know-how of the knowledge gained [6,7].

The SECI process spearhead activities that enable the creation, transfer, and utilisation of knowledge acquired from the $\mathrm{Ba}$. The tacit and explicit are substituted and changed based on circumstance and environment. It transforms knowledge from conceptualisation (theory) to actualisation (practice) [6]. Knowledge assets emanate from $\mathrm{KC}$ that connect to humans and technology. It happens via socialisation of knowledge, sharing experiences from tacit. Externalisation, voicing tacit to explicit; combinationstructuring explicit; and internalisation-applying new knowledge learned from explicit to tacit with conducive environment and leadership support [1]. Knowledge creation and sharing are the institution's limelight that forms the knowledge management strategy for sustainable development $[14,15]$. Knowledge is about knowing via socialisation.

\subsection{3 $\mathrm{Ba}$}

$\mathrm{Ba}$ as a "shared space for an emerging relationship". This could be in an office, "bantaba" (community gathering in villages), market, street, or social media to share ideas and experiences [7]. Information and knowledge acquired from $\mathrm{Ba}$ are chaotic, unstructured, and difficult to communicate for understanding. Thus, it is a platform to share context that enables and stimulates new knowledge creation $[6,7,16,17]$

\subsubsection{Knowledge Assets (KAs)}

Knowledge assets are the inputs, outputs, and moderating factors of the knowledge-creating process. As a result, KAs encompass the content of the knowledge creation process. Assets are specific resources that are essential and worthy for disaster risk management and disaster risk reduction in disaster management institutions. Despite this, KAs are intangible, tacit and dynamic. And difficult to capture, but the result is from achievements made from performance capabilities. Knowledge assets are in two forms: human (soft) skills, experiences, norms, values, and IT (hard) technology for codification, storing, collection, and retrieval [18]. These components form the effective operations of an institution.
$\mathrm{KC}$ highlighted four types of knowledge assets: Experiential KAs, Conceptual KAs, Systemic KAs, and Routine KAs. The research found that the $\mathrm{KC}$ process consists of $\mathrm{Ba}$, SECI, and KAs. In utilising the existing knowledge assets, new knowledge is created via the SECI process, which energises $\mathrm{Ba}$.

\subsection{Knowledge creation (KC) in DRM/DRR}

Knowledge Creation is a new discipline in the $\mathrm{DRM} / \mathrm{DRR}$, and very few research materials are available. Disaster risk management and disaster risk reduction require sufficient knowledge creation and sharing for the institution's operations.

Disaster risk management, reduction, and knowledge creation are relevant in any given situation of development and growth. For the past years, the progress in knowledge and use of DRM/DRR knowledge is slowly coming up in disaster operations. This is because Priority three of the Hyogo Frameworks for Action (HFA) 2005-2015 emphasised the use of knowledge, innovation, and education in DRM/DRR [19]. It also stated in the Sendai Framework for Disaster Risk Reduction (SFDRR) 2015-2030 priority one [20], understanding disaster risk, and priority three investing in DRR for resilience (HFA 2005-2015 and SFDRR 2015-2030) are essential. Knowledge creation influences individuals and the environment from interaction and exceeding beyond the boundary to seek knowledge.

Some articles highlighted knowledge and creation as the most relevant source for competitive strength. Thus, the institution initiates and delineates bottlenecks and cultivates and uses new knowledge to solve the SECI problem. KC has not been applied and impacted to institution's growth between top management and knowledgeable workers and partner institutions in DRM/DRR [6,7,16,17]

Knowledge significantly contributes to DRM/DRR, which supports $\mathrm{KC}$ and its influence on the institution and is positively impacted. Disaster risk management and reduction researchers revealed that DRM/DRR requires interaction with authority, groups, workforce, resources, partnerships, and systems to avoid failure rather than boost the competitive environment [7]. $\mathrm{KC}$ in DRM/DRR “enables mixing and matching solutions across different disasters" for leadership to monitor and coordinate disaster events [21]. The Focus was more on knowledge management which focuses on the institution knowledge such as acquisition, accumulation, use, and reuse of present knowledge, abandoning the DRM/DRR knowledge creation process to give a chance to new knowledge from the tacit. Instead, the shift was mainly on explicit knowledge.

So far, from the few kinds of research, $\mathrm{KC}$ is evolving intensely in many knowledge management institutions. Notwithstanding, there are gaps in KC, such as epistemological, which is a different understanding of 
types of knowledge relevant to the institution. The strategic component looks at the lack of understanding of the skill and expert knowledge on DRM/DRR, whereby the institutional gap focuses on how institutions absorb and transfer knowledge and the right human resource. These four gaps are cross-cutting for the operationalisation and implementation of $\mathrm{DRM} / \mathrm{DRR}$. Despite the gaps, $\mathrm{KC}$ is becoming increasingly important in all DRM/DRR strategy and policy angles to do away with the old fashion of doing things differently to achieve better performance [22].

\subsection{Leadership}

Leadership in disaster risk management and risk reduction $\mathrm{KC}$ is central because they provide the environment, are proactive, creative, and learn beyond the boundary for better improvement. The leadership has a role in $\mathrm{KC}$ by defining, redefining, or developing a knowledge vision to establish knowledge assets or the existing knowledge assets to create new knowledge of the institution through the SECI process from the $\mathrm{Ba}$. The knowledge created becomes part of the institution's knowledge assets. Leadership covers all the aspects of DRM/DRR coupled with leading knowledge vision promoting, sharing, creating, energising $\mathrm{Ba}$ and continuous development of knowledge spiral of $\mathrm{KC}$ from central and distributed (top and middle) leadership by reading the situation and leading to the three elements of $\mathrm{KC}$ [23].

Through leadership questioning on "What are we?", "What should be created?", "How can we do it?", "Why are we doing this?" and "Where are we going?" will determine and monitor the institution knowledge creation from the past knowledge that is limitless and to give new knowledge for development and retention with the involvement of top and middle management and the staff in the $\mathrm{KC}$ process fostering commitment to the $\mathrm{KC}$ process [7]. Central and distributed leadership increases the institution effectiveness coupled with a focusoriented investing in skills development, structures in place, and technology that will create cultural learning system [23]. Thus, KC is up-to-date by an institution vision outlined informing leadership on strategic objectives [5,7]. The leadership should always take urgent decision making during an emergency, even sometimes when there is no information at that particular moment but applying critical thinking for action is necessary.

The SECI connects with positive leadership as the leader is the coordinator, director, facilitator, and evaluator of programmes and achievements [23]. There is no one size fit in disaster risk management and disaster risk-reduction leadership rather proactive actions to reduce the situation impact accordingly. In this case, some studies employed strategic leaderships, which constitutes the institution's strategy, policy formulation, vision, mission, fostering culture, and knowledge creation platform. Leaders at the apex of decision-making should not underrate the power of knowledge creation, which lacks the entrustment of care, trust, love, support, and concern in promoting human development at all levels in advancing growth [23].

As far as there is a new paradigm shift of $\mathrm{KC}$ from the traditional sense of "management" controlling the flow of information from a top-down approach rather "middle-up-down" approach to the knowledge creation process has to be recommended [7]. The knowledge creation process facilitated by middle-up management leadership to take ownership of the three fundamentals of $\mathrm{KC}$ as a vision to inform and know the types of knowledge assets required for operationalisation through a self-internal assessment to attain a knowledge vision.

Leadership impacted the institution's effectiveness by formulating strategy, vision, mission and fostering institutional culture. Therefore, strategic leadership in SECI relates to motivating, inspiring, and empowering staff coupled with a conducive environment [23]. The research focused on strategic leadership to help shape institutional development and growth because of the multidimensionality of the institutions and will reduce back and forward line ministry decision-making bureaucracy. Therefore, understanding the role of leadership can influence leadership perceptions [24].

The management of institutions needs to bank on knowledge to generate a plan for justifiable development in disaster risk management and disaster risk reduction. Disasters are emerging with complexity due to extreme climate events. Globalisation is increasing with natural hazards and anthropogenic activities deterring social-economic development and loss of lives in all aspects. Changes in information technology are constant and diverse. Therefore, leadership in KC's strategic leadership believes that applying the current dynamic environment to enhance institutional effectiveness in DRM/DRR is important and timely. On the contrary, leadership bureaucracy also hinders the institution's operationalisation [25]. The dimension of leadership eases the smooth running of the institution.

\section{Methodology}

The research adopted a qualitative case study and content analysis approach to analyse the data using Voyant tools looking at the subject and concept of the KC SECI process in NDMA. The method applied for assessment gives the researcher the ability to comprehend the informant's perspectives. During this process, the validity and reliability of the data matter. Stratified random sampling was applied for NDMA and partners for the data collection. And Five (5) informants were selected from NDMA with two senior central staff from administration, finance, and three regional 
coordinators Lower River Region, West Coast Region, and Upper River Region. The chosen staff have experiential knowledge of the DRM/DRR issues in The Gambia and a long working relationship with the NDMA from its inception to the research period. They are involved in DRM/DRR implementations and operations at central and regional levels with knowledge of on-the-job training. They helped to have access to information and feedback during the data collection within the institution and partners.

Open-ended questionnaires were drawn and administered through the use of Google Forms electronic questionnaire. This electronic questionnaire gives easy accessibility to informants to answer at their own suitable time. Four (4) questions targeting NDMA but during the process to have more information and balancing the data, institution partners co-opted. Eight (8) institutions with one (1) representative identified and including NDMA gives a total of nine (9) institutions taking part as in Table 1 .

Table 1. Targeted institutions for the assessment of KC

\begin{tabular}{|c|c|c|}
\hline No & Institution/Informants & Total \\
\hline 1 & NDMA & 5 \\
\hline 2 & Gambia Red Cross Society & 1 \\
\hline 3 & National Environment Agency & 1 \\
\hline 4 & Gambia National Nutrition Agency & 1 \\
\hline 5 & Gambia Bureau of Statistic & 1 \\
\hline 6 & Ministry of Health and Social Welfare & 1 \\
\hline 7 & Ministry of Agriculture & 1 \\
\hline 8 & Village Development Committee & 1 \\
\hline 9 & University of Gambia & 1 \\
\hline
\end{tabular}

Partners chosen are members of the National Platform for DRR with comparative advantages in financing, expertise \& technical knowledge, equipment, infrastructure, leadership style, and institutional culture for being proactive in their respective fields. They are multi-sectoral national platform committees established to help central coordination, advice, and analysis on priority areas for action to the central government towards its national development plan formulations, implementations, and the overall achievement of the Sustainable Development Goals for disaster management issues. The synopsis of these partners' institutions is below.

\section{Result}

The Act of parliament mandated NDMA to coordinate disaster management issues in the Gambia. The Vice President is the overall supervisor and the chairperson of the disaster governing council, and the Executive Director works in close liaison to multifaceted institutions to implement disaster activities in building a resilient country. There are decentralised structures at regional and municipal, district, and village levels under the purview of Governors and Mayors, Chiefs, and Alkalos.

Disaster risk management and disaster risk reduction require sufficient knowledge and knowledge creation for the institution's operations and implementation. For the past years, the progress in knowledge and use of DRM/DRR knowledge is slowly coming up in disaster operations. There are gaps in NDMA KC, such as epistemological, human resource, strategic, institutional, and financial commitment. Despite the gaps, $\mathrm{KC}$ is becoming increasingly important in all DRM/DRR strategy and policy angles to do away with the old fashion of doing things differently to achieve better performance [22]. The research has shown that SECI has been unnoticed and uncoordinatedly implemented in NDMA "Happy Corner" and the National Platform for DRR. SECI unearthed NDMA's strengths, weaknesses, opportunities, and threats. There is a lack of leadership support and commitment for $\mathrm{KC}$ whereby no or limited resources are budgeted for staff growth. Fear factors building capacities would lead to coup d'état of positions by juniors who acquired knowledge and new knowledge for a competitive environment.

\subsection{Knowledge creation process in NDMA}

\subsubsection{Knowledge}

According to informants, knowledge is a critical motivation for people. Knowledge has to be shared, which gives information and enhances capacity versavisa. As a result, information is communicated, including ideas and research, to establish institutions' vision, mission, and objectives for operations. In this case, knowledge surfaced 23 times. It means that knowledge is relevant to NDMA and partners, as the quotes below by the informants.

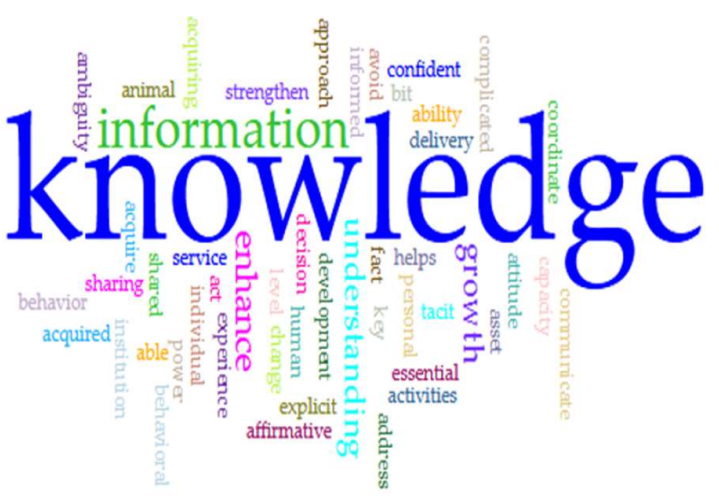

Fig. 2. Knowledge in relation to the KC process of SECI in NDMA 


\subsubsection{Knowledge creation (KC)}

The KC code and frequency count 11 times. They further mentioned its importance to bridge the knowledge gap of an institution through information, experience, and sharing formally or informally from the interactions. Knowledge creation in DRM/DRR promotes staff and community resilience as drivers to sustainability to development and economic health by reducing poverty.

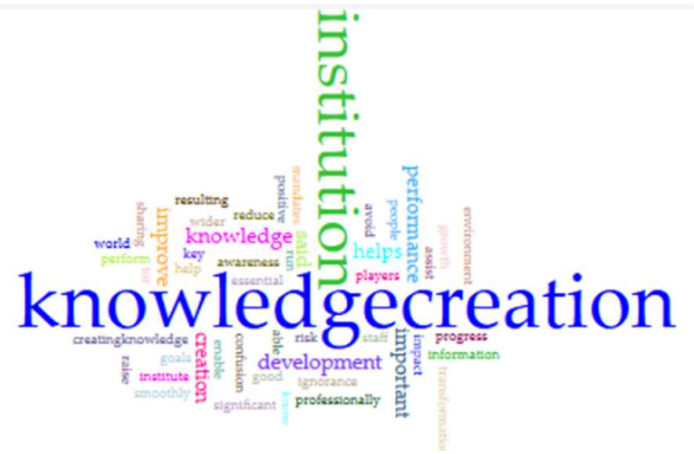

Fig. 3. Knowledge creation in NDMA

\subsubsection{SECI}

The explanation and understanding of SECI are in tandem with a literature review of the research, which influences individuals and the environment. It is from interaction and exceeding beyond the boundary. It can be learning, training, apprenticeship from the institution, traditional home, and community. Informants further mentioned SECI could be formal and informal consultations, commitment, and political will. The most frequent word in the corpus is SECI 34 times.

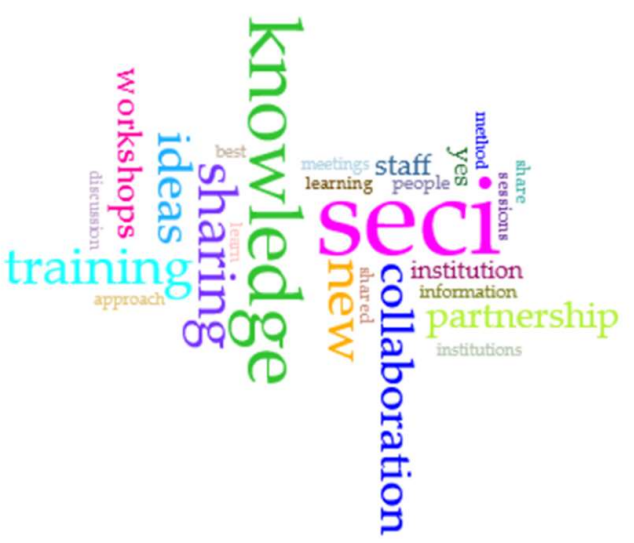

Fig. 4. SECI in NDMA

As a result, the framework developed and modified is relevant to informed decision-making in the $\mathrm{KC}$ process as leadership in the centre enables SECI of the institution.

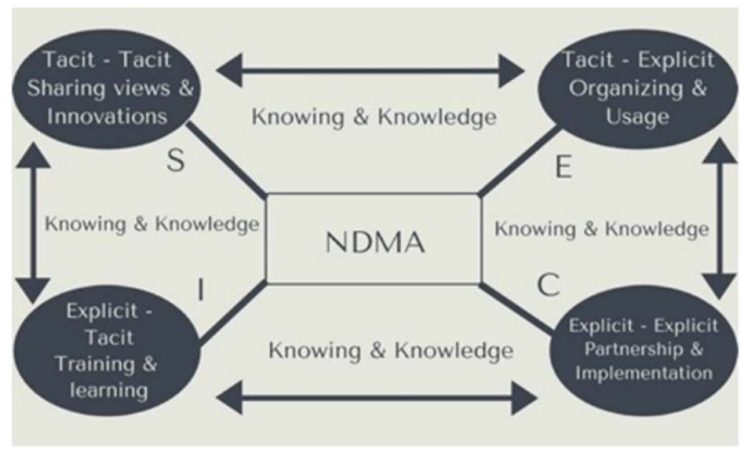

Fig. 5. SECI framework adapted and developed during the assessment Nonaka et al. (2000) modified by Mbassi Sanneh 2021

The framework developed will serve as a guide to influence and strengthen NDMA's revised strategy and policy. The relevance of knowledge creation through the use of knowledge, innovation, education, and investment.

\subsubsection{Socialisation, originating $\mathrm{Ba}$ and experiential knowledge asset.}

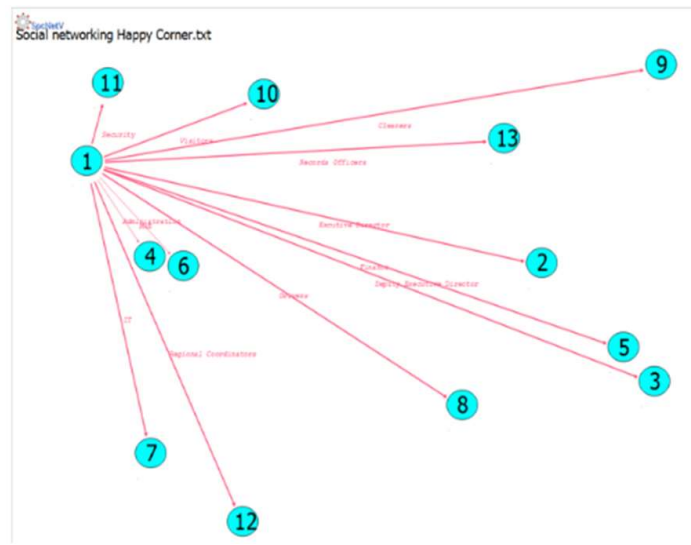

Fig. 6. "Happy Corner" networking in NDMA

The "Happy Corner" (internal socialisation) is an opportune place that enables knowledge growth where dialogue on tacit knowledge emanates from sharing experiences unsystematically at NDMA [26,27].

\subsubsection{Externalisation, interacting $\mathrm{Ba}$ and conceptual knowledge asset}

The collaboration and communication with a group of people - partners to create knowledge. NDMA works with multidisciplinary institutions on disaster risk management and disaster risk reduction, which led to the establishment of the National Platform for disaster risk reduction (DRR) for networking which includes government ministers, private sectors, academic 
institutions, Red Cross Society, Religious leaders, NGOs, civil society, media, and UN agencies. This platform is a multidisciplinary stakeholder national structure that helps to advocate DRR at all levels. They are responsible for coordination, advice, and analysis on priority areas for action. Success national platform depends on national ownership and leadership of the DRR process [28]. The partners have comparative advantages on financing, knowledge, expertise, equipment, infrastructure, leadership style, and institutional culture for being proactive in dealing with DRM/DRR based on the component such as resource mobilisation, advocacy, coordination, and technical committees. The National Platform for DRR was established and officially declared in March 2011 as a national committee for DRM/DRR. It was a piloted project in the Gambia for other West African countries to learn from best practices with funding and partnership support from ECOWAS. They aim to create a partnership structure that will influence political and legal commitment for DRM/DRR understanding and strengthen the scientific knowledge of DRM/DRR governance among the public and authorities and mainstream it to the national development plan for sustainable development of the country.

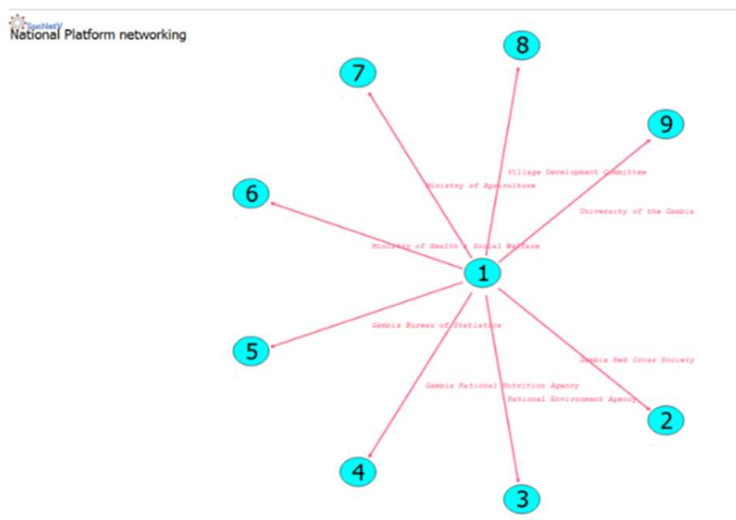

Fig. 7. The national platform for DRR networking in NDMA

\subsubsection{Combination, systemic Ba and systemic knowledge asset}

Combination of ideas from the National Platform for DRR minutes, reports, policy, strategy, or work plans to systematically codify for respective institutional plans aligned with international frameworks for DRM/DRR [29].

In this process, leadership must base on a shared understanding of DRM/DRR with the multi-sectoral and multidisciplinary structure to actively facilitate central and distributed leadership for all partners' involvement and participation for a better combination of concepts on ideas and suggestions the National Development Plan for sustainable development. Partners involved in planning concepts and implementing demonstrations from the SECI to transfer knowledge [25] through leadership support for $\mathrm{KC}$ for a better result. The system is more structured and efficient for learning, sharing plans, policy, and strategy to improve decision-making. It helps in capacity building and easy retrieval of documents and records keeping. The combination is processed through partnership support to NDMA in leveraging knowledge from existing knowledge and KAs to plan for improvement. Tacit to explicit knowledge transform to create skill and experience via combination for staff use and institutions. If not, the knowledge will gradually disappear in NDMA and partner institutions without proper documentation and record-keeping. The National Platform for DRR is the platform for the SECI, more so the externalisation and combination through interaction and collaboration with partner institutions to implement DRM/DRR activities regardless of comparative advantages.

\subsubsection{Internalisation, exercising $B a$ and routine knowledge asset}

In NDMA, learn-on-the-job, simulation exercises, training of trainers on comparative expertise skills, and internship to learn new knowledge for better operations of the institution are semi-dormant because the leadership is not investing in KC. Secondly, partner institutions are having similar challenges. The platform is where partners learn new knowledge through socialisation and practice during interactions to domesticate explicit knowledge to their tacit knowledge for internal implementation. This is not happening due to the fear factor as in the quotes.

Thereby, the NDMA's existing knowledge mapped out surfaced new knowledge availability after reviewing the SECI process that energises the "Happy Corner", National Platform, and KAs at NDMA [1,7,17]. The mapping supported NDMA leadership to know the competencies and capacities of NDMA and partners. It helped identify and define the knowledge vision relevant to the agency, and equally, communication and accessibility are genuine in KAs [30].

\subsection{Role of leadership in KC process in NDMA}

\subsubsection{Leadership}

Creating the environment to implement creativeness and ideas from lessons learned beyond the boundary is for better improvement. Therefore, leadership support creating the environment to implement lessons learned beyond for better improvement is relevant. Leaders define, redefine, and develop existing knowledge assets to create new knowledge from the SECI process. Notably, leadership covers all the aspects of DRM/DRR coupled with leading knowledge vision promoting, sharing, creating, energising $\mathrm{Ba}$ and continuous 
development of knowledge spiral of $\mathrm{KC}$ from central and distributed (top and middle) leadership by reading the situation and leading to the three elements of $\mathrm{KC}$ $[7,23]$.

The knowledge vision defines the type of knowledge required for an institution to create. Through leadership questioning, "What are we?", "What should be created?", "How can we do it?", "Why are we doing this?" and "Where are we going?" Therefore, leadership constitutes strategy, policy formulation, vision, mission, fostering culture, and knowledge creation processes.

Leadership connects to Ba, SECI, and KAs as the three elements of the $\mathrm{KC}$ process. The report also mentioned that primary responsibility in DRM/DRR relies on strong leadership. In Fig. 8, the keyword is highlighted 30 times and emphasised by informants how leadership can facilitate $\mathrm{KC}$ for the growth of any institution, as mentioned below.

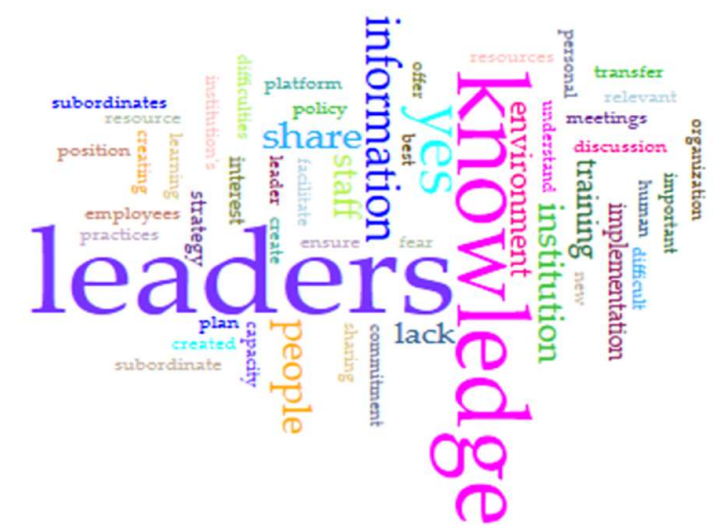

Fig. 8. Leadership role in $\mathrm{KC}$ process in NDMA

Table 2. Summary of leadership dimensions in NDMA KC

\begin{tabular}{|l|l|}
\hline Condition & Leadership \\
\hline Collaboration & Interactions with transparent leadership is weak. \\
\hline Beliefs & $\begin{array}{l}\text { "Fear factor" to be challenged by staff when } \\
\text { capacities are built. }\end{array}$ \\
\hline Process & $\begin{array}{l}\text { Learning best practices and accept critics of } \\
\text { practicing and formalising the theory to a situation. }\end{array}$ \\
\hline Authority & "Information is monopolised". \\
\hline Skills & $\begin{array}{l}\text { Leadership powers are not utilise at central and } \\
\text { distributed leadership. }\end{array}$ \\
\hline Development & $\begin{array}{l}\text { Formulate strategic issues and develop programmes. } \\
\text { Investing in skills, development, structures in place, } \\
\text { and technology create a cultural learning system. }\end{array}$ \\
\hline
\end{tabular}

\section{Discussions}

$\mathrm{KC}$ process is fragmented in NDMA and not coordinated between humanitarian workers and skilled workers. Therefore, there is a gap in information sharing and coordination in DRM/DRR. Lack of this could be an inadequate performance of current DRM/DRR operations in The Gambia. KC is essential in DRM/DRR to learn from lessons and adopt best practices for skills improvement and enhance knowledge creation by building a culture of learning from pre, during, past, and future hazards and disasters. Through KC, adequate knowledge can be accessed and utilised at all levels by empowering and protecting staff and the institution as a whole. "Ignorant is no excuse to the law". The research discovered that NDMA and partners have experiential knowledge.

As mentioned above, decision-making and timesensitive are very dynamic in DRM/DRR operations. Therefore, a single delay in dissemination would negatively impact the decision, which will affect embracing the KC process in NDMA DRM/DRR. When the know-how is there, it makes a fast decision for action in all operations for better achievement. So, therefore, $\mathrm{KC}$ is a gateway for knowledge acquisition and sharing through interdisciplinary contacts by developing shared understanding towards a common purpose. KC reinforces the working practices and allows learning and applying new knowledge in the work systems. Nevertheless, despite the hidden KC process in NDMA and its partners, the researchers noticed that the SECI was implemented in an uncoordinated manner both internally and externally without leadership becoming aware of and not knowing the framework for $\mathrm{KC}$ and its relevance. Finally, for the implementation of $\mathrm{KC}$ to be effective, the role of middle management is to interpret and communicate the $\mathrm{KC}$ process horizontally and vertically through leadership interactions in meetings, conferences, or any place of $\mathrm{Ba}$ for learning.

There are numerous barriers to $\mathrm{KC}$ that hinder the effective knowledge creation of an institution that needs to uninstall obstacles of lack of leadership support and discouragement, trust, care, and love. There is a lack of transparency and accountability by the leadership. For example, within NDMA, programmes or information will not reach individuals concerning the matter; instead, you hear it from hearsay. Such would inspire discouragement, lack of trust, care, and love for socialisation in the working environment and hindered performance delivery for growth. Also, bottlenecks are lack of the know-how to tap the unnoticed knowledge creation process, information sharing on time, bureaucracy, capacity building, poor infrastructure, resistance to change, and implementation of the words into actions.

Furthermore, the human factor could be barriers, lack of individual self-confidence, fear of criticism, bias concerning strict orders, and obsolete systems and procedures of an institution that can deter KC of NDMA and partners. Inadequate information sharing, interaction, and communication monopolised. Research results, "trained human resource" and financial commitment are currently challenging in the country DRM expressed by staff. To avert such challenges, they recommended "staff and volunteer training" to enhance the capability to achieve NDMA goals.

The political interference in the operations of NDMA malnourished the management and leadership 
style as the central office mandated to coordinate all disasters in the country. Parse politics have no direct implications on DRM/DRR but rather on institutional formation, management, operations, and laws governing the institution. It is high time to revisit and unattached humanitarian operations from political affairs to effectively and transparently implement according to national, regional, and international guidelines irrespective of political affiliation and tribe. Disaster risk management and disaster risk reduction should not be influenced or dominated by politics.

Resonate from the beginning discussing the research benefit NDMA knowing the $\mathrm{KC}$ process available from the proposed framework SECI easy implementation. The leadership can achieve its vision to protect and build resilient communities with appropriate knowledge and generally strengthen the growth of the agency and partners for efficient and effective DRM/DRR operations and implementations. Research paper for knowledge management to be conducted by NDMA using this research for reference as the baseline assessment.

\section{Conclusion}

There should be continuous improvement in performance by leveraging $\mathrm{KC}$ and striving towards $\mathrm{DRM} / \mathrm{DRR}$ to achieve the institution's objectives. KC will enhance operational excellence and leadership commitment. NDMA and partners need KC theory for successful implementation to address the four stages of knowledge conversion. It will identify tacit and explicit knowledge needs and the current condition of the $\mathrm{KC}$ process and provide leadership with good guidance (proposed framework) that would contribute to achieving NDMA objectives through SECI. HFA 20052015 and SFDRR 2015-2030 country assessment report mentioned why the framework objectives are not attainable according to the time frame due to capacity constraints of countries in the implementation of priorities. The Gambia contributes to the delay capacity constraints of right human resources, strong leadership, and finance.

The KC process is transcending individual boundaries for new knowledge to create through the SECI process. Therefore, the assessment highlighted the underlying factors and explored issues affecting the current condition of $\mathrm{KC}$ in NDMA and partner institutions. The current condition of the $\mathrm{KC}$ process on SECI has been unnoticed and uncoordinatedly implemented in NDMA "Happy Corner" and the National Platform for DRR and the new spiral of knowledge creation.

The leadership is weak with frequent consultation to the vice president's office and leads to NDMA bureaucracy in operations, implementation, and frequent leadership changes by the previous and current government. There are leadership constraints for capacity building with no or limited resources budgeted. There is no specific budgetary allocation for DRM/DRR $\mathrm{KC}$ and even for the whole DRM/DRR like in other countries advocating for a certain percentage of the national budget investment to DRM/DRR programmes. The only allocation for NDMA is the emergencies response account. Another challenging issue at hand is that NDMA and many other agencies are under the purview of the vice president. And the DRM/DRR projects can be influenced by politics by other players in DRM/DRR field. There is a lack of commitment, support, lacking self-confidence, and fear factor to build staff capacity that would lead to coup d'état positions by juniors. The unwillingness to cope with new knowledge, inadequate or sometimes no information sharing, interaction, and communication monopolised. In a nutshell, NDMA leadership is a challenge with institutional management, financial, and knowledge creation.

\section{References}

1. Afri Consultan Group, Needs Assessment for Disaster Risk Management, Recovery Planning and Checklists for Preparedness in the Face of Climate Risks (2017)

2. CADRI, Disaster Risk Reduction Capacity Assessment Report, the Gambia (2012)

3. D. Amaratunga and R. Haigh, Post-Disaster Reconstruction of the Built Environment (WileyBlackwell, Oxford, UK, 2011)

4. P. Akhavan, S. Ghojavand, and R. Abdali, Journal of Information \& Knowledge Management 11, 1250012 (2012)

5. C. Bratianu, I. O.-M. \& Marketing, and undefined 2010, Core.Ac.Uk (n.d.)

6. I. Nonaka, I. o Nonaka, N. Ikujiro, and H. Takeuchi, The Knowledge-Creating Company: How Japanese Companies Create the Dynamics of Innovation (1995)

7. I. Nonaka, R. Toyama, and N. Konno, Long Range Planning 33, 5 (2000)

8. D. Bennet and A. Bennet, VINE 38, 72 (2008)

9. A. Rahman and K. Munadi, in IOP Conference Series: Earth and Environmental Science (IOP Publishing, 2019), p. 12040

10. A. Rahman, A. Sakurai, and K. Munadi, International Journal of Disaster Risk Reduction 29, 13 (2018)

11. A. Rahman, A. Sakurai, and K. Munadi, in $I O P$ Conference Series: Earth and Environmental Science (IOP Publishing, 2017), p. 012018

12. Fachrurradhi, N. R. M. Susanti, A. Pratama, R. Fitri, S. Raudhiah, A. Rahman, and K. Munadi, in IOP Conference Series: Earth and Environmental Science (IOP Publishing, 2019), p. 12043 
13. K. North and G. Kumta, Knowledge Management : Value Creation through Organizational Learning (Springer, 2014)

14. P. Akhavan, S. Ghojavand, and R. Abdali, Journal of Information and Knowledge Management (2012)

15. R. K. and F. Bulutlar, The Influence of Knowledge Sharing on Innovation (2010)

16. I. Nonaka, Organization Science 5, 14 (1994)

17. I. Nonaka and H. Ayano, Social Innovation Creating New Knowledge for New Social Value (Graduate School of International Corporate Strategy, 2010)

18. K. Seneviratne, D. Baldry, and C. Pathirage, International Journal of Strategic Property Management (2010)

19. IRIDeS, "Hyogo Framework for Action 2005 2015: Building the Resilience of Nations and Communities to Disasters" HFA IRIDeS Review Report Preliminary Focusing on 2011 Great East Japan Earthquake (Sendai, 2013)

20. UNISDR, The Sendai Framework for Disaster Risk Reduction 2015-2030 (2015)

21. S. H. Othman and G. Beydoun, ACIS 2010 Proceedings (2010)

22. K. Albris, K. C. Lauta, and E. Raju, International Journal of Disaster Risk Science 11, 1 (2020)

23. G. von Krogh, I. Nonaka, and L. Rechsteiner, Journal of Management Studies 49, 240 (2012)

24. J. Hartley and L. Rashman, in Connecting Knowledge and Performance in Public Services, edited by K. Walshe, G. Harvey, and P. Jas (Cambridge University Press, Cambridge, n.d.), pp. $145-172$

25. E. C. K. Cheng, in (2015), pp. 11-23

26. M. L. Farnese, B. Barbieri, A. Chirumbolo, and G. Patriotta, Frontiers in Psychology (2019)

27. J. H. Song, D. Uhm, and S. W. Yoon, Leadership and Organization Development Journal (2011)

28. ISDR, UNISDR Informs: Disaster Risk Reduction in Asia \& Pacific (2006)

29. J. Hoon Song, D. Uhm, and S. Won Yoon, Leadership \& Organization Development Journal 32, 243 (2011)

30. B. Marr, G. Schiuma, and A. Neely, International Journal of Business Performance Management 4, 279 (2002) 